\title{
Les forêts vosgiennes à l'épreuve des tempêtes sous l'Ancien Régime (XVIIe et XVIIIe siècles)
}

The Vosgian Forests tested by storms under the Old Regime (17th-18th Centuries)

Die Wälder der Vogesen in den Stürmen des 17. und 18. Jahrhunderts

\section{Emmanuel Garnier}

\section{(2) OpenEdition}

\section{Journals}

\section{Édition électronique}

URL : http://journals.openedition.org/rge/4114

DOI : $10.4000 /$ rge.4114

ISSN : 2108-6478

Éditeur

Association des géographes de l'Est

Édition imprimée

Date de publication : 1 juin 2000

ISSN : 0035-3213

Référence électronique

Emmanuel Garnier, "Les forêts vosgiennes à l'épreuve des tempêtes sous l'Ancien Régime (XVIle et XVIIIe siècles) », Revue Géographique de l'Est [En ligne], vol. 40 / 3 | 2000, mis en ligne le 03 août 2013, consulté le 08 septembre 2020. URL : http://journals.openedition.org/rge/4114 ; DOI : https://doi.org/ $10.4000 /$ rge. 4114

Ce document a été généré automatiquement le 8 septembre 2020.

Tous droits réservés 


\title{
Les forêts vosgiennes à l'épreuve des tempêtes sous l'Ancien Régime (XVIIe et XVIIIe siècles)
}

\author{
The Vosgian Forests tested by storms under the Old Regime (17th-18th \\ Centuries) \\ Die Wälder der Vogesen in den Stürmen des 17. und 18. Jahrhunderts
}

\section{Emmanuel Garnier}

\section{Introduction}

1 Interrogé à propos de l'ampleur de la tempête du 26 décembre 1999, l'historien Emmanuel Le Roy Ladurie a répondu sans aucune hésitation que « la France n'avait jamais connu une telle catastrophe ${ }^{1}{ }^{1}$. Ce jugement, plutôt expéditif, apparaît prématuré car le dépouillement des sources de l'administration forestière d'Ancien Régime, notamment celles concernant les Vosges, conduit à tempérer fortement cette déclaration prononcée sous le choc de l'événement. L'approche historique, parce qu'elle s'opère sur le temps long et qu'elle prend en compte de manière globale les contextes social et spatial, permet à la fois de relativiser l'impact de la dernière tempête et de tirer des enseignements que le gestionnaire serait bien mal avisé de négliger pour l'avenir.

2 Fondé sur l'analyse des archives émanant des anciennes institutions forestières qu'étaient les grueries $^{2}$ et les maîtrises ${ }^{3}$, le présent travail se propose d'esquisser l'histoire des catastrophes météorologiques ayant touché les forêts vosgiennes situées aujourd'hui dans le cadre du territoire du Parc Naturel Régional des Ballons des Vosges. Cet exposé, il ne faut pas le cacher, pose davantage de questions qu'il ne résout les problèmes concrets d'actualité. Une telle situation s'explique essentiellement par la nature de sources, souvent imprécises et incomplètes, qui laissent une large place à l'extrapolation et à la subjectivité. 
3 Dans ces conditions, il faudra s'interroger sur le phénomène "tempête " afin de déterminer ce qu'il recouvre exactement sous l'Ancien Régime et s'il s'agit d'un événement exceptionnel ou si, au contraire, il représente une donnée permanente pour la forêt vosgienne. L'étude de la fréquence des catastrophes est surtout destinée à dégager, si elles existent, des périodes cataclysmiques plus marquées dont l'impact catastrophique pour les écosystèmes forestiers vosgiens s'expliquerait pour partie par l'action anthropique exercée sur le milieu.

\section{Les phénomènes météorologiques anciens}

\section{A. « Chablis » et « volis »}

Les archives forestières n'abordent la question des bois victimes des vents qu'au travers de mentions laconiques faisant référence à des «chablis » et à des «bois dépéris » ce qui certes, autorise une approche historique mais qui, malheureusement, incite l'historien à une certaine extrapolation. Celui-ci est en effet confronté au redoutable écueil qu'est le sens à donner à ces mots, d'autant plus que jamais les sources n'opèrent de franche distinction entre les « chablis » et les « volis ».

5 Le caractère polysémique de ces termes trouve un début d'explication à la lecture de $\mathrm{M}$. Chailland, auteur du «Dictionnaire raisonné des Eaux et Forêts» (1769), référence obligée pour tout historien de la forêt. Dans cet ouvrage, l'action des vents n'est abordée qu'avec le mot «chablis» dont la définition, très générale, désigne «tous les arbres abattus par les vents ou par quelqu'autre accident d'origine naturelle » (M. Chailland, 1769). Ainsi, le forestier d'Ancien Régime semble confondre sous une même appellation à la fois les sujets abattus ou renversés et ceux cassés au niveau du tronc que l'on nomme aujourd'hui « volis ». Ce n'est qu'un demi-siècle plus tard que les deux principaux types de dégâts engendrés par les vents font enfin l'objet d'une distinction chez M. Baudrillart. Son « Traité général des Eaux et forêts » désigne les chablis comme étant des arbres «abattus, renversés, arrachés ou rompus au pied par les vents, ou tombés de vieillesse ou de pourriture, ou par le poids des neiges». Pour la première fois, l'ouvrage aborde la question des « volis » ou « volins », des sujets brisés à la moitié ou aux deux tiers de leur hauteur ou bien encore ceux dont les branches maitresses ont été arrachées (M. Baudrillart, 1823). Ainsi, l'interprétation historique qui suit reste tributaire de l'imprécision des sources émanant de l'administration des Eaux et Forêts d'avant 1789.

\section{B. Les chablis de la première moitié du XVIIle siècle (Fig. 1)}

6 Les registres des ventes de bois de la gruerie de Bruyères sont une précieuse source d'information pour l'historien forestier puisqu'ils consignent les bois « chablis » ayant fait l'objet de ventes au profit du domaine ducal et des chanoinesses de Remiremont, propriétaires indivis des forêts lorraines du massif ${ }^{4}$. Dans les années 1740 , les chablis ne représentent guère que $11,5 \%$ des bois vendus, généralement à des prix très faibles, la moyenne se situant autour de 20 sols le pied alors que le prix habituel d'un arbre vif est de l'ordre de 5 livres $^{5}$. Pour l'année 1740 par exemple, le revenu procuré par la commercialisation des arbres chablis ne représente qu'un peu moins de $7 \%$ des recettes totales. Ce type de source est riche d'enseignements car, souvent, est indiqué le 
nom de la forêt dont sont originaires les arbres abattus. Un dépouillement minutieux et systématique de ces registres, non réalisé à ce jour, permettrait probablement de cartographier les forêts les plus sujettes aux chablis pour la période prérévolutionnaire.

Fig. 1 : Cadre géographique et localisations

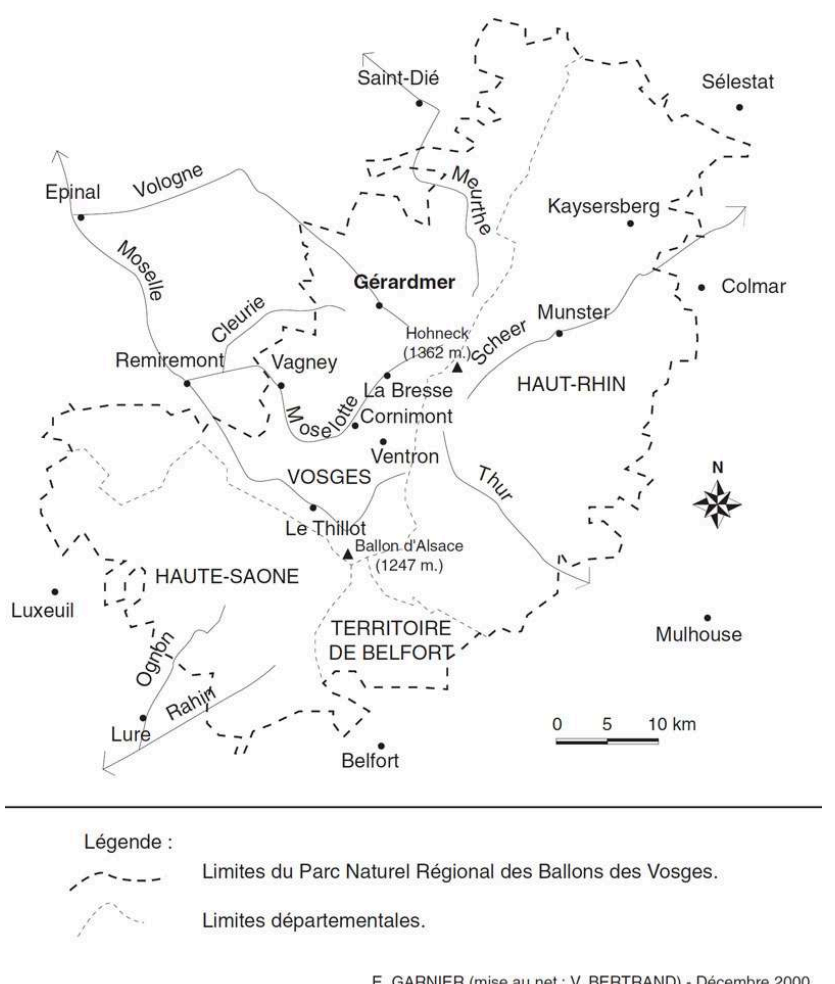

7 Il serait néanmoins erroné de déduire de ces différentes observations que les bois chablis représentaient peu de choses dans le cas de la gruerie de Bruyères au milieu du XVIIIe siècle. En réalité, une part importante de ces bois de faible valeur est, à cette époque et conformément à un usage traditionnel, consommée sous la forme de bois $\mathrm{d}^{\prime}$ affouage ${ }^{6}$. Et, de fait, les règlements forestiers donnés par les autorités ducales entre le XVIIe et le XVIIIe siècle le prouvent amplement. Celui de 1764, promulgué pour aménager les forêts de la montagne ordonne aux officiers de la maitrise d'Epinal de ne délivrer de bois vif aux usagers pour chauffage qu'après avoir employé à cet effet les "chablis et autres arbres tombés et de nulle valeur " ${ }^{7}$. Mais il ne s'agit là que de prescriptions officielles qui ne correspondent pas forcément à la réalité sylvicole du terrain c'est pourquoi il est indispensable de recourir à un autre type de données d'archives : les registres de délivrance des bois d'affouage et de maronage ${ }^{8}$.

Véritable mine d'or pour qui s'intéresse aux pratiques usagères ainsi qu'à l'état des écosystèmes forestiers montagnards, ces documents ont l'intérêt de recenser précisément le nombre et l'état sanitaire des arbres voués au chauffage et aux constructions des paysans vosgiens. Les essences les plus couramment em-ployées pour approvisionner les foyers sont le sapin et le hêtre, ce qui correspond assez logiquement aux peuplements dominants dans les Vosges, c'est-à-dire à la hêtraie-sapinière. Il semble bien avoir pléthore d'arbres chablis comme l'atteste l'exemple de la communauté de Ventron en 1728. Les autorités seigneuriales n'ont même pas besoin de marquer d'arbres car ils en trouvent suffisamment pour l'affouage «arrachés par les 
vents ». Dans les années 1780, le volume de bois de bâtiment délivré aux habitants du ban de Vagney se compose, pour $22 \%$, de bois chablis (E. Garnier, 2000). Il faut signaler au passage que l'exploitation de ces arbres sous l'Ancien Régime, comme encore de nos jours, restait très dangereuse pour le bûcheron, toujours à la merci d'un accident. Les levées de cadavres contiennent ainsi plusieurs exemples de professionnels victimes des chablis comme ce fut le cas pour Joseph Bernard de Jussarupt qui, alors qu'il sciait au "passe-partout» un sapin renversé, fut emporté par les racines alors que le tronc glissait ${ }^{9}$.

\section{Les tempêtes de la seconde moitié du XVIIle siècle}

La seconde moitié du XVIIIe siècle marque un temps de rupture par rapport à la période antérieure dans la mesure où les chablis habituels, fruits des rigueurs du climat montagnard, font place à de véritables catastrophes naturelles dont l'impact sylvicole est autrement plus dramatique. Désormais, les archives parlent de plus en plus fréquemment de « tempêtes » et d'" ouragans ", autant d'accidents climatiques majeurs qui semblent avoir particulièrement impressionné les témoins de l'époque.

Le mouvement est inauguré par les forêts de la vallée de Munster victimes à deux reprises, en 1741 et 1746 , d'une « tempête » s'accompagnant de violents coups de vents dévastateurs pour la sapinière puisque des milliers d'arbres sont couchés ou brisés. Les événements se déchainent de nouveau en 1750 dans la forêt comtoise de Saint-Antoine et trois ans plus tard dans les forêts communales de Gérardmer avec, à chaque fois, un même paysage de désolation forestière. A compter des années 1760, les forêts vosgiennes sont victimes, selon un rythme décennal, d'«ouragans", comme en témoignent les forêts de Kaysersberg, touchées de plein fouet par un ouragan qui ravage une grande partie des bois de la cité, les sources indiquant que plusieurs milliers d'arbres ont été déracinés. En 1770, c'est au tour de Gérardmer et des communautés proches de subir les assauts d'un nouvel ouragan particulièrement bien décrit par les habitants ${ }^{10}$. Là, une très forte tempête s'accompagnant de vents tourbillonnants se déplace sur une longue distance selon une trajectoire courbe qui sinistre une dizaine de villages. Les arbres des forêts communales sont déracinés par les vents puis emportés par un torrent de boue charriant aussi des roches et les sols agricoles. Quatre ans plus tard, les orages de l'été se révèlent tout aussi dévastateurs car la forêt résiduelle en marge des finages est devenue très vulnérable et une fois de plus les arbres paient un lourd tribut.

11 Ces catastrophes météorologiques majeures, apparemment inconnues jusque-là, méritent sans doute d'être mises en relation avec les grandes tendances climatiques du XVIIIe siècle. L'historien Emmanuel Le Roy Ladurie (1983) observe bien une certain " attiédissement» du climat au cours de la décennie 1740 qui tranche singulièrement avec les rigueurs de la fin du XVIIe siècle. La tendance au réchauffement est telle qu'il parle même de "vague chaude " pour les années 1757-1763 et 1770-1780 et il est bien tentant d'établir une corrélation entre cette évolution et les ouragans que connaissent les Vosges sensiblement au cours des mêmes années.

Ainsi, loin d'être un phénomène inconnu, les coups de vents restent une donnée permanente dans l'histoire des forêts vosgiennes et les archives démontrent que les bois chablis ou volis constituaient un aspect non négligeable de la gestion des espaces ligneux en fournissant le bois indispensable à la survie du montagnard. Cependant, les 
années 1740-1750 semblent inaugurer de nouvelles décennies où les phénomènes météorologiques tendent à prendre l'allure de catastrophes naturelles au sens où nous l'entendons aujourd'hui. Les conséquences forestières négatives de ces tempêtes ne peuvent guère s'expliquer qu'à l'aune des contextes sylvicole et humain.

\section{L'impact des fronts pionniers sur les écosystèmes forestiers vosgiens}

\section{A. Essor des fronts pionniers au XVIIle siècle (Fig. 1 et 2)}

A compter du début du XVIIIe siècle, les communautés rurales du massif vosgien connaissent un essor démographique exceptionnel qui se traduit par une vague de défrichements sans précédent culminant après 1770 . Les assauts de l'homme contre l'arbre se déroulent sur deux fronts, le premier part des vallées pour atteindre l'étage montagnard, tandis que le second se situe aux étages montagnard supérieur et subalpin, où les «marcaires" ne cessent d'empiéter sur le domaine forestier qui régresse là aussi ${ }^{11}$.

Fig. 2 : Défrichements et ouragan : la région de Gérardmer en 1770.

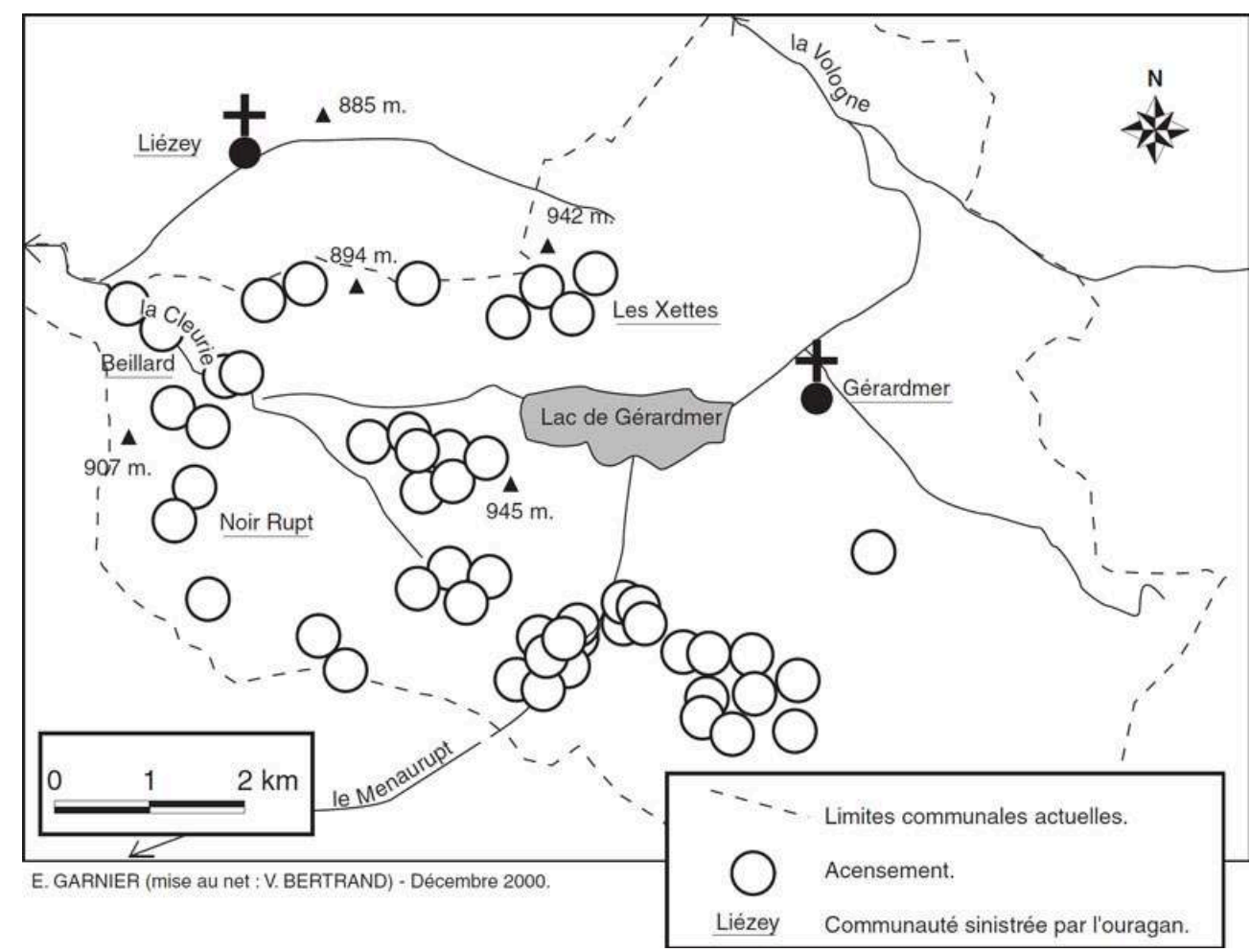

Dans la périphérie immédiate du village, les plus humbles font reculer les bois communaux désignés dans les sources sous le terme de "rapailles» en créant des essarts et des "fouillies». Les premiers concernent des défrichements de surfaces réduites mais fort nombreuses, voués le plus souvent à la culture de la pomme de terre, tandis que les secondes sont une forme originale de mise en valeur temporaire des sols forestiers. Pratique culturale très répandue dans les Vosges, la fouillie consiste à raser une portion de forêt d'une surface moyenne de 50 ares pour ensuite brûler la parcelle 
déboisée et la labourer. La terre ainsi enrichie par les cendres de la strate herbacée est alors prête à être semée en seigle ou en pomme de terre. Le feu joue donc un rôle important puisqu'il contribue à nettoyer radicalement le terrain des plantes adventices et de leurs graines ainsi que des parasites. Mais il favorise aussi l'enrichissement organique, à l'origine de la diminution de l'acidité du sol et de la mobilisation d'éléments fertilisants dans les cendres (E. Garnier, 2000).

L'acensement, quant à lui, permet au paysan de faire reculer la sapinière de l'étage montagnard. Vecteur essentiel de la colonisation de la montagne, l'acensement est une pratique juridique originale par laquelle le seigneur se dépouille d'une partie de son domaine, à savoir une portion du sol forestier, au profit d'un sujet qui lui verse une redevance et acquitte les droits seigneuriaux. Le censitaire est réellement propriétaire de son immeuble puisqu'il peut en disposer à sa guise, à condition d'observer les clauses de son contrat, à savoir la résidence et le versement d'un cens. Dans les années 1720, la vallée de la Haute-Meurthe comptait plus de 180 acensements disséminés un peu partout dans les forêts de l'abbaye de Saint-Dié. Avec une moyenne de 4 hectares par cense, ce sont plus de 700 hectares de bois qui auraient été défrichés dans cette seule partie des Vosges lors du premier tiers du XVIIIe siècle. L'écrasante majorité des acensements est implantée au cœur des forêts et ces espaces ouverts enclavés génèrent un manteau forestier mité aux contours flous. Ces foyers de colonisation ouvrent des fronts pionniers multiples et très dynamiques.

Les chaumes enfrichées ou reboisées après le terrible épisode guerrier du XVIIe siècle connaissent à partir des années 1720 un vigoureux mouvement de remise en valeur (E. Garnier, 2000). Destinés à accueillir les bovins des vallées, ces pâturages d'estive situés au niveau de la crête sont autant de foyers de déboisement. A la veille de la Révolution, les grandes chaumes sommitales accueillent un troupeau de l'ordre de 1000 animaux, soit une très forte charge pastorale. Le prélè- vement ligneux opéré dans les forêts limitrophes se fait sous trois formes essentielles. La première est la quête du combustible menée en permanence par le pasteur afin de chauffer son lait pour la préparation $\mathrm{du}$ fromage. La seconde, omniprésente alors, est le combat quotidien engagé contre l'arbre pour agrandir la chaume. Mais le tribut payé par l'arbre ne s'arrête pas là car il doit aussi subir l'assaut des bovins dévorant indistinctement la strate herbacée du sous-bois et les jeunes pousses. A titre indicatif, il faut savoir qu'au cours de son séjour sur la chaume qui dure environ quatre mois, le marcaire maintient un feu continuel pour chauffer son lait. A la veille de 1789 , la consommation ligneuse globale des chaumes sommitales peut être estimée à un peu moins de 1000 stères, un nombre qui ne tient pas compte du volume de bois employé par les petites chaumes ouvertes clandestinement. Or, les chaumes secondaires créées au cours de cette période pour la seule vallée de Munster s'élèvent à 215, soit un gouffre engloutissant annuellement près de 3440 stères (E. Garnier, 2000)!

\section{B. Conséquences sur les forêts}

17 Le dynamisme des fronts pionniers dont la particularité vosgienne est d'être multiforme et dispersée engendre une fragilisation de la sapinière bien visible au travers du dépouillement des procès verbaux de visites rédigés à partir des années 1750. 
18 Ainsi, les inspecteurs chargés de visiter les forêts de la vallée de Munster en 1758 font très régulièrement allusion à une pratique couramment utilisée par les marcaires pour défricher : le surcenage ou kerben en Alsace. Cette technique de défrichement encore usitée par les colons sibériens au XIXe siècle sous le nom d'ocercivat' est une permanence à l'échelle du massif vosgien, à l'origine d'une riche toponymie (Cerneux, La Cercenée, Kerboltzkopf). Le surcenage consiste à pratiquer une entaille sur la circonférence du tronc à l'aide d'une hache, au printemps, lors de la montée de sève. Quelques années plus tard, ce n'est plus qu'une rangée d'arbres secs sur pied qui entourent la chaume et il n'est pas difficile alors de les abattre car la méthode fait obstacle à la multiplication des rejets, ce qui favorise une extraction aisée des racines. Aussi n'est-ce pas surprenant de constater dans les rapports que nombre de hêtres ou de sapins sont victimes de cette pratique dont les effets ne tardent pas à se faire sentir. Dans le canton du Steinberg (massif du Hohneck) par exemple, les forestiers observent, impuissants, qu'un grand nombre d'arbres ont été abattus par les vents dans un secteur récemment cerné par les bergers locaux.

19 La multiplication des acensements provoque des dégâts encore plus conséquents. Dans la forêt royale de Gérardmer, il est possible d'en recenser une vingtaine qui sont autant de foyers de dégradation totalement incontrôlables. Les périmètres forestiers touchés par les nouvelles vagues de création de censes des années 1720 sont " défigurés » en raison des vides occasionnés par les défrichements et la divagation des bêtes. Fragilisée dans sa structure par les trouées qui y ont été réalisées, la sapinière est la victime toute désignée des coups de chablis et des bris de neige qui ne font encore qu'amplifier son recul. Les conséquences dramatiques de l'ouragan de 1770 dans la région de Gérardmer semblent directement imputables à la déforestation engendrée par les acensements. La comparaison de la carte des censes réalisés sur le territoire gérômois au XVIIIe siècle avec les localités sinistrées révèle, de manière très probante, une corrélation parfaite entre les deux composantes (Fig. 2). C'est en suivant la vallée de la Cleurie que les défrichements ont proliféré sur les hauteurs dominant les villages. L'ouverture de ces enclaves, prélude au déboisement total de versants entiers, a sans doute joué un rôle majeur en amplifiant les effets dévastateurs de l'ouragan du mois de juillet 1770.

\section{La remise en cause de la sylviculture traditionnelle}

\section{A. Le jardinage traditionnel vosgien}

Jusqu'au milieu du XVIIIe siècle, la sapinière vosgienne fait l'objet d'une gestion relativement rationalisée. Le règlement de la prévôté de Bruyères, en 1620, consacre la reconnaissance par le pouvoir central d'une sylviculture ancestrale adaptée aux écosystèmes montagnards : le jardinage. Loin de pouvoir être réduit à une sylviculture de cueillette, le jardinage préconisé par ce texte est fondé sur une intervention sylvicole réfléchie consistant à extraire prioritairement les arbres de petites dimensions afin de rajeunir le groupe des jeunes sujets et de dégager les plus beaux arbres. Il contribue aussi à maintenir des structures étagées et irrégulières formant des ensembles-tampons mieux à même de résister aux phénomènes météorologiques (E. Garnier, 1999).

21 La sapinière située à l'étage montagnard offre, à partir du témoignage des archives, un paysage forestier d'un grand intérêt se caractérisant par une forte biodiversité. La forêt 
se présente comme un espace mouvant, mal défini, se caractérisant par une profonde imbrication des milieux ouverts et fermés. Impliquant une gestion de classes d'âges diversifiées et le maintien de strates hétérogènes, l'intervention de l'homme passe d'abord par une limitation des surfaces de coupe, gage d'hétérogénéité spatiale à échelle réduite. Les autres modalités de cette sylviculture extensive se fondent aussi sur le maintien de niches écologiques et paysagères comme les arbres mal conformés, les clairières et les tourbières, la conservation de très vieux arbres, autant de particularités favorables à la flore et à la faune. La futaie décrite par les sources apparaît très claire et le mélange d'essences beaucoup plus riche. Les sapins sont ainsi mélangés aux hêtres et pins sylvestres, mais aussi à des érables planes dans les parties les plus élevées, bien éclairées (E. Garnier, 1998). Il ne semble donc pas exagéré de dire que le jardinage vosgien ancestral a permis, jusqu'aux années 1750 au moins, de conserver une dynamique plus naturelle favorable au maintien de biotopes remarquables au regard des critères écologiques actuels. Dans une contribution récente, l'écologue J.C. Rameau (2000) ne manque pas d'ailleurs d'observer que les futaies de sapin des Vosges, bien qu'endommagées, demeurent des forêts dignes de ce nom, ce qui est loin d'être le cas pour les forêts régulières monospécifiques, peu ou mal éclaircies.

\section{B. Le « tire et aire » des forêts royales et ses conséquences}

22 Le royaume de France fait alors figure de parent pauvre de la sylviculture de montagne car la science forestière royale a complètement oublié les forêts résineuses situées sur les terres du Roi Très Chrétien. Confinée dans une routine sylvicole dont la composante majeure est le "tire et aire", un mode d'exploitation éprouvé dans les bassins de la Seine et de la Loire, la politique forestière française ignore tout de la sylviculture des résineux ${ }^{12}$.

23 Pressé par la demande ligneuse des arsenaux, de plus en plus dépendants des importations des pays de la Baltique, Colbert édicte un règlement global en 1669. Il impose un régime sylvicole unique pour l'ensemble du royaume, mettant du même coup hors la loi l'ancestral jardinage vosgien. Si le «tire et aire » favorise le chêne, essence de lumière capable de tolérer un brutal découvert, il engendre en revanche de nombreux inconvénients pour le hêtre ou le sapin, des espèces appréciant particulièrement l'ombre et une croissance juvénile sous couvert.

Le paradoxe majeur réside donc dans le fait qu'un texte initialement destiné à assurer la conservation des forêts se révèle totalement inadapté, voire dangereux pour les écosystèmes montagnards. La coupe rase paraît ainsi dévastatrice dans les sapinières et les pessières, ces deux essences ne rejetant pas de souche mais se régénérant au contraire à partir de semences. De plus, la brutale exposition des sujets conservés dans les coupes exploitées les condamne immanquablement en raison de la faible profondeur de leurs racines traçantes. Malgré les avertissements des enquêteurs royaux dépêchés sur place peu après la conquête, les autorités royales ont préféré faire prévaloir le modèle centralisateur français sur les versants alsacien et comtois des Vosges.

Dans ces conditions, la partie méridionale du massif ne tarde pas à être touchée par de violents coups de chablis dont la chronologie et le bilan ont précédemment été évoqués à partir du contexte climatique. Dans la vallée de Munster, toutes les conditions sont 
réunies pour que les tempêtes de 1741 et 1746 soit désastreuses. Dans les deux cas, les forêts ont été fragilisées après que l'abbé, puis le Magistrat de Munster y aient introduit la méthode du taillis sous futaie. Les exploitants du moment, conformément aux règlements, ont rasé la totalité des arbres peuplant les coupes, à l'exception de quelques sapins de futaie conservés comme arbres de réserve (baliveaux) en vue de la régénération de la sapinière. Mais cette précaution s'avère insuffisante face aux rigueurs du climat vosgien. Quelques mois plus tard, les propriétaires constatent, impuissants, l'étendue du désastre. Qu'il s'agisse des versants alsaciens ou comtois, le scénario reste le même, les vents violents s'engouffrent dans les assiettes (coupes) brutalement découvertes sur lesquelles ne subsistaient que les arbres réservés, immédiatement couchés. Le bilan est lourd puisque la régénération de la forêt est directement mise en péril, faute de semenciers en mesure de pérenniser la sapinière d'origine.

Le paysage de désolation offert par les forêts couvertes de bois chablis est vécu comme un véritable choc psychologique par les communautés locales, peu habituées à un tel gâchis des ressources forestières. C'est pourquoi une prise de conscience se fait jour à l'échelle du massif, pour évoluer progressivement vers un refus pur et simple de la politique sylvicole imposée par la monarchie. Au-delà d'une simple résistance de populations autochtones au pouvoir central, le mouvement traduit plus sûrement la revendication d'une foresterie ancestrale reposant sur l'emploi du jardinage.

\section{Conclusion}

Cet exposé, malgré des limites certaines liées au contenu des sources disponibles, prouve néanmoins qu'il est possible d'appréhender d'une manière relativement fiable l'histoire des tempêtes aux XVIIe et XVIIIe siècles à travers le filtre forestier vosgien. Pour mener à bien ce type d'enquête, l'indicateur "chablis ", même si le terme recouvre plusieurs sens sous l'Ancien Régime, constitue un choix pertinent pour l'historien de la forêt. Loin d'être un phénomène exceptionnel, les arbres victimes des vents sont au contraire un facteur de permanence dans les forêts vosgiennes d'antan, ce qui ne peut constituer une surprise pour le gestionnaire forestier actuel.

Cependant, le recul historique pointe du doigt quelques pistes pouvant apporter des éléments d'explication quant à l'ampleur plus ou moins grande de l'impact des catastrophes météorologiques sur les écosystèmes forestiers. Si la problématique des fronts pionniers n'est plus d'actualité, le massif étant davantage menacé par la reconquête de l'arbre aujourd'hui, celle abordant la question de la sylviculture est probablement la plus féconde pour les institutions confrontées au problème de la reconstitution des espaces ligneux. La forêt vosgienne traditionnelle, composée d'une futaie claire, multistrate et bien mélangée était ainsi mieux à même de donner aux peuplements une plus grande résilience. A l'avenir, les pouvoirs publics en charge du dossier «tempête » seraient bien mal inspirés de ne pas envisager un volet historique dans leur réflexion d'ensemble car, comme l'a dit l'historien J. Le Goff «L'histoire ne donne pas de leçons, ni ne prédit l'avenir, mais elle éclaire les choix ». 


\section{BIBLIOGRAPHIE}

"Après les tempêtes ", $\mathrm{n}^{\circ}$ spécial de la revue de l'ONF Arborescences, $\mathrm{n}^{\circ}$ 84, mars-avril 2 000, pp.

$2-46$.

Baudrillart M. (1823). - Traité général des Eaux et Forêts. Tome 1, Paris : Bertrand-Huzard, 1006 p.

Chailland M. (1769). - Dictionnaire raisonné des Eaux et Forêts. Tome 1, Paris : Ganeau et Knapen, $621 \mathrm{p}$.

Dralet E. (1820). - Traité des forêts d'arbres résineux et des terrains adjacens sur les montagnes de France. Toulouse : Vieusseux, $271 \mathrm{p}$.

Laroussinie O., Drouineau S. dir. (2000). - Expertise collective sur les tempêtes, la sensibilité des forêts, et sur leur reconstitution. Rapport GIP-ECOFOR, INRA, CEMAGREF, $22 \mathrm{p}$.

Le Roy Ladurie E. (1983). - Histoire du climat depuis l'an mil. Paris : Flammarion, 287 p.

Garnier E. (1998). - Un massif forestier et son histoire : la forêt de Saint-Antoine (permanences, mutations et enjeux). Paris : Office National des Forêts, 137p.

Garnier E. (1998). - Jalons pour une histoire de l'environnement : la Réserve Naturelle du FrankenthalMisseimle. Relations des sociétés et du milieu. Munster : Parc Naturel Régional des Ballons des Vosges, $59 \mathrm{p}$.

Garnier E. (1999). - « Le jardinage traditionnel dans les Vosges : une sylviculture proche de la nature avant l'heure (XVIe-XVIIIe siècle) ? ». Colloque international « La sapinière », Parc Naturel Régional des Ballons des Vosges (La Bresse 28-29 septembre et 1er octobre 1999). Sous presse.

Garnier E. (2000). - « The coveted tree : the industrial threat to the Vosges Forest in the 16th and 18 th centuries ». In M. Agnoletti, S. Anderson. Forest History. International studies on socioeconomic and forest ecosystem change. IUFRO, Wallingford : CABI Publishing, tome 2, pp. 37-47.

Garnier E. (2000). - « Survivre en montagne sous l'Ancien Régime. Le rôle de l'affouage dans le massif vosgien (XVIe-XVIIIe siècle) ». Journée d'études Environnement, forêt et société « Le bois énergie », Paris : IHMC, ENS Ulm, CNRS, cahier d'études nº 10, pp. 9-18.

Garnier E (2000). - Les forêts des Vosges méridionales à l'époque moderne. Des espaces éclatés et convoités. Thèse d'histoire, Université de Besançon, 795 p. A paraître aux presses de l'office National des Forêts.

Hotto H.J. (2000). - « Expériences sylvicoles après des ouragans catastrophiques. Regards dans le passé en Basse-Saxe ». Nancy, Revue Forestière Française, n 3, pp. 223-238.

Husson J.P. (1995). - Les forêts françaises. Nancy : Presses Universitaires de Nancy, 258 p.

Husson J.P. (1997). - « Les paysages anciens de la forêt domaniale de Gérardmer, contribution patrimoniale à la révision d'aménagement ». Nancy, Revue Forestière Française, n 5, pp. 469-476.

Rameau J.C. (2000). - « Ecosystèmes et tempêtes ». Les écosystèmes forestiers dans la tempête, Paris, GIP-ECOFOR, 101p. pp. 119-127.

Rottmann M. (1986). - Wind und Sturmschäden im Wald. Beiträge zur Beurteilung der Bruchgefährdung, zur Scha-densvorbeugung und zur Behandlung Nadelholzbestände. Frankfurt-amMain, 1285 p., 1986. 
Schutz J.P. (1997). - Sylviculture 2. La gestion des forêts irrégulières et mélangées. Lausanne : Presses polytechniques et universitaires romandes, 1997, $178 \mathrm{p}$.

\section{NOTES}

1. Le Monde du 7 janvier 2000.

2. Gruerie : juridiction forestière subalterne sous l'Ancien Régime.

3. Maîtrise des Eaux et Forêts : juridiction forestière supérieure sous l'Ancien Régime. Elle était dirigée par un maître particulier qui siégeait à Epinal.

4. Arch. dép. Vosges, G 2300, registre, Vente de bois de la gruerie de Bruyères. Fonds du chapitre de Remiremont.

5. 1 livre $=20$ sols .

6. Les registres de ventes des bois mentionnent bien le montant des recettes procurées par les bois d'affouage, malheureusement ils n'indiquent pas la nature des arbres en question, à savoir s'il s'agit de sujets renversés ou brisés par les vents.

7. Arch. dép. Vosges 3 E $55 n^{\circ}$ 2086, Arrêt du Conseil portant règlement des coupes ordinaires dans les forêts de la montagne (10 mars 1764).

8. Le bois de maronage désigne les arbres délivrés aux usagers comme matériau de construction

9. Arch. dép. Vosges, 3 B 210, Levées de cadavres de la prévôté de Bruyères (28 octobre 1741).

10. Arch. com. Gérardmer, DD $10 \mathrm{n}^{\circ}$ 2, Supplique des habitants de Gérardmer au sujet d'un ouragan (13 août 1770).

11. Marcaire : terme désignant le préposé à la garde des troupeaux et à la confection des fromages. Le berger séjournait plusieurs mois sur les pâturages d'estive appelés « chaumes » dans les Vosges.

12. Tire et aire: exploitation sylvicole réalisée de proche en proche du taillis-sous-futaie composé de feuillus.

\section{RÉSUMÉS}

L'étude historique de l'impact des tempêtes et des ouragans sur les forêts vosgiennes aux XVIIe et XVIIIe siècles peut être conduite à partir des archives forestières de l'époque. Les chablis, loin d'être exceptionnels, sont une composante habituelle des paysages forestiers anciens. Cependant, à partir des années 1740, ils tendent à se multiplier en raison d'accidents climatiques majeurs. Deux facteurs essentiels peuvent alors être mis en cause, la colonisation et la sylviculture. La première fragilise la forêt en y réalisant des défrichements ponctuels tandis que la seconde, en généralisant une politique forestière inadaptée, contribue à rendre les peuplements plus vulnérables qu'auparavant.

The historical study of the impact of storms and hurricanes on Vosgian forests in the 17th and 18th Centuries can be made from forest archive of the period. Uprooted trees, far from being exceptional, are a normal component of old forest landscapes. However, since 1740, they have multiplied because of major climatic accidents. Two essential factors can be held responsible, colonisation and forestry. The first weakens the forest by making clearings while the second, a 
generally unsuitable forest policy, contributes to making the populace more vulnerable than before.

Die historische Untersuchung der Auswirkung von Winden und Orkanen auf die Wälder der Vogesen im 17. und 18. Jahrhundert kann mit Hilfe der damaligen Forstarchivalien durchgeführt werden. Die keineswegs aussergewöhnlichen Windwürfe sind ein übliches Merkmal früherer Waldlandschaften. Indessen nehmen sie seit 1740 als grössere Klimaschäden immer mehr zu. Zwei Hauptfaktoren sind dabei wesentlich: die Besiedlung und der Waldbau. Erstgenannte schwächt den Wald durch punktuelle Rodungen, während letztgenannter mit einer generellen, nicht angepassten Forstpolitik dazu beiträgt, die Waldbestände anfälliger als früher zu machen.

\section{INDEX}

Mots-clés : chablis, défrichement, jardinage, ouragan, sapinière, sylviculture, taillis sous futaie, tempête, Vosges, XVIIe et XVIIIe siècles

Keywords : 17th and 18th Centuries, clearing, coppice with standards, fir forest, hurricane, selection system, silviculture, storm, uprooted trees, Vosges

Schlüsselwörter : 17. und 18. Jahrhundert, Forstwirtschaft, Mittelwald, Orkan, Plenterung, Rodung, Sturm, Tannenwald, Vogesen, Windwurf

\section{AUTEUR}

\section{EMMANUEL GARNIER}

Université de Franche-Comté - Faculté des Lettres et Sciences Humaines - 30, rue Mégevand 25030 Besançon Cedex 